(pp 21-27)

\title{
Association between Very Early Initiation of Rehabilitation and Rehabilitation Provision System for Stroke Patients: Analysis According to Day of Admission
}

\author{
Daisuke MATSUMOTO, PT, MS \\ Department of Physical Therapy, Faculty of Health Science, Kio University
}

Katsunori KONDO, MD, PhD

Center for Well-being and Society, Nihon Fukushi University

Nariaki SHIRAISHI, PT, MS

Department of Rehabilitation, Faculty of Health Sciences, Nihon Fukushi University

Motoya SUGIYAMA, PT, MS

Department of Rehabilitation, Chubu Rosai Hospital

Seungwon JEONG, PhD

Department of Social Science, National Center for Geriatrics and Gerontology

Purpose: To investigate the association between very early initiation of rehabilitation (VEI) and a rehabilitation provision system for acute stroke patients by performing an analysis according to the day of admission.

Methods: Data of stroke patients ( $\mathrm{n}=2,307 ; 8$ hospitals) from the Japan Rehabilitation Patients Database in Japan were examined. The patients were divided into the following five groups according to the day of admission: (1) weekday with no holidays (Monday to Thursday), (2) weekday with a holiday, (3) Friday, (4) Saturday and Sunday, and (5) holiday. We defined VEI as rehabilitation commencing within 3 days of stroke admission. Multiple logistic regression analysis was used to evaluate VEI, involvement of rehabilitation specialists as an attending doctor, and the ratio of rehabilitation staff members to beds.

Results: We found that VEI for acute stroke patients was significantly associated with involvement of a rehabilitation specialist and a high ratio of rehabilitation staff members to beds $(\mathrm{p}<0.05)$.

Conclusion: Our results suggest that when providing VEI for acute stroke patients, it may be effective to promote the involvement of a rehabilitation specialist as an attending doctor, and increase the number of rehabilitation staff members. 